\title{
Technology and Human Skills in Manufacturing
}

\author{
H.-H. Erbe \\ Technische Universität Berlin \\ Sekr. FR 4-4 - Franklinstrasse 28/29 \\ D-10587 Berlin, Germany
}

\begin{abstract}
To be competitive SME's with small batch production or tool- and mould making are forced to use numerical controlled manufacturing processes and computer based shopfloor control. But the technological support for these enterprises should be shopfloor oriented to foster and develop human skills in flat hierarchies avoiding unnecessary dividing of work. With shopfloor oriented production support human skills and automation create synergetic effects. The mastery of the manufacturing process is in the hands and brains of skilled workers at the shopfloor. Automation gives the necessary support to execute tasks and rationalize decisions. This represents a way of low cost automation. To run the manufacturing process effectively is not only a question of technology though an essential one. Together with an adequate work organization wherein human skills can be developing themselves it establishes the frame for a competitive manufacturing in SME's.
\end{abstract}

\section{INTRODUCTION}

Technologies which support the skill of the workers but not replace it are called shop floor oriented. We may define some criteria:

1) in respect to the man-machine interfaces:

-flexible machine tools with easy access to the working area

-manual and numerical controllable machine tools

-graphical-interactive programmable NC-controls as well as PC-software

-multimedia supported interpretation of the machine diagnosis in respect to maintenance by operators

2) in respect to machine-machine interfaces:

-at the shopfloor usable PC's with NC-programming software for different controls but with identical structures and user surfaces as these machine's tool controls and with program transfer between machine controls and PC in both directions

3) in respect to fine planning

-conventional planning boards or shopfloor control systems usable by skilled workers at the shopfloor for decisions in respect to scheduling tasks, shorten the through-put time and solving problems caused by break-downs of machines, missing tools or jigs and fixtures, material or personnel..

4) in respect to produce high quality

-devices for in-process measuring

-for all skilled workers usable statistical process control

-at the shopfloor usable coordinate measuring machine 
Group- or Teamwork supporting technology is an additional criteria. This means the technology should be usable in a sporadic manner by all skilled workers depending on their tasks just now. This avoids the necessity of specialists. On the other hand should the workorganization just hinder the cultivation of specialists, because these are not favourable for small and medium sized enterprises.

\section{CONVENTIONAL AND NUMERICAL CONTROLLED MACHINE TOOLS}

Using numerically controlled machine tools is until now mostly divided in planning/programming the necessary steps to manufacture parts, the setting up of tools, clamping devices as jigs and fixtures and the operating of the machine tools. These tasks are given to different personnel like engineers, technicians and skilled workers. This is based on the usual tayloristic dividing of work, supported by the thinking of engineers to avoid qualified human intervention to the manufacturing process at the shopfloor if even possible. An example is the $\mathrm{CAD} / \mathrm{CAM}$ process chain, i.e. planning and programming the manufacturing more or less automatically at the CAD-screen far away of the workshop. Then usually problems arises as the engineers or technicians at the $\mathrm{CAD}$ have not enough direct experiences with running the technological process of manufacturing at the machine tools and the operators have difficulties to intervene into the sophisticated programming, which is very often necessary.

Company Traub [1] was the first builder of machine tools for complete processing in one machine (turning, milling, drilling) avoiding alpha-numeric programming. The user surface is very near to handling the machine in a conventional way. It uses pictogramms representing the processes like cutting the contour, roughing or finishing the surface, to select chucks and tools, offers feed rates and cutting velocities depending on the material. The operator has to fill in datas, selects a certain process, sets markers for synchronization and the programming to control the CNC-axis is running in the background (Fig. 1 and Fig. 2).

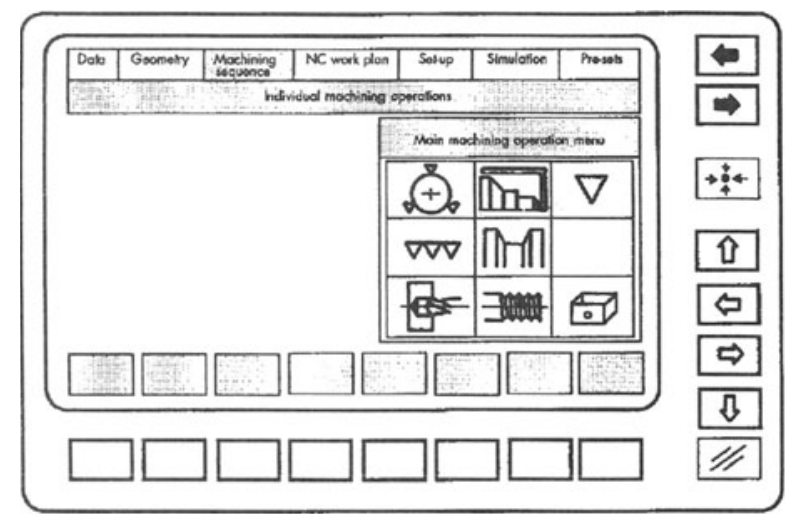

Fig. 1. User Surface of Traub-Control

But nevertheless the operator stays away from the direct manufacturing process. Simulation is possible of course. It gives a very good information on the planned manufacturing process. But the software only allows processes structured before within the software. So far the operator is running his tasks partly blind, restricted to handling software. Despite all advantages compared to usual controls, the experience of the workers are not properly used. 


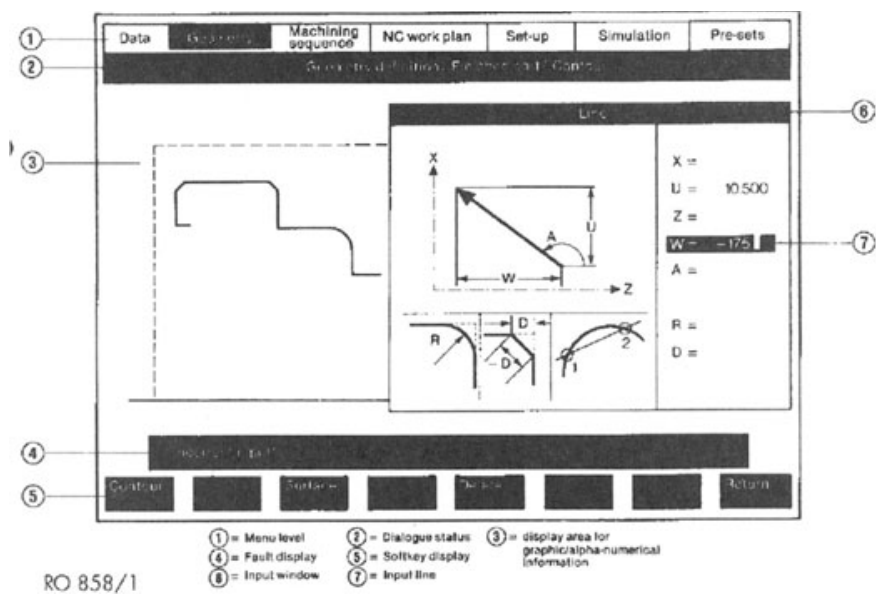

Fig. 2. Example for Data Input at Traub-Control User Surface

Recently new controls got developed allowing for handling numerically controlled machines manually via handwheels or joysticks and if necessary software support may be added. This is more than the well known teach-in and play-back as will be explained later. CAD datas may be used of course avoiding the time consuming manual input of geometry from drawings. But all the technology of the manufacturing process is in the hands (and brains) of the skilled workers at the shopfloor. They may use the machine tool conventionally with handwheels thereby mobilizing their direct experiences or they may get software support wherever this is appropriate. The intelligent software supervises all movements and knows the exact position of the tools at any time. If the geometry was defined before, the software prevents violations of the contour even in the manual mode.

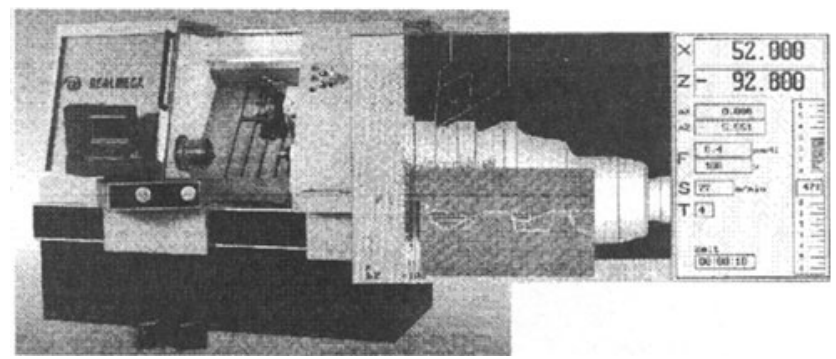

Fig. 3. Example of Keller/NUM-Control at a Machine Tools for Turning

Companies Keller/NUM [2] uses a concept wherein the handling/operating of a machine tool for turning with handwheels gives the operator a more direct access to the manufacturing process (Fig. 3). Four modes are possible: manual, semi-automatic, automatic, automatic with intellegent software support (Fig. 4): 
1. manual: handwheels for two axis are used together with a combined one for chamfers. The operator works like at a conventional machine.

2. semi-automatic: the manufacturing steps are generated manually using the handwheels but immediately simulated at the screen before or simutaneously with the processing. Software support is provided if necessary.

3. automatic: the planning/programming of the manufacturing will be done off-line at the screen of the control or using the same software running at a PC. Pictogramms are provided to support the programming, no alpha-numerical sentences are necessary. The technological datas are added when first the geometry of the part has been defined. CAD may be connected.

4. automatic with software support: optimal tool pathes, recognizing changed raw part geometry, workplan optimization, preventing of contour violation.

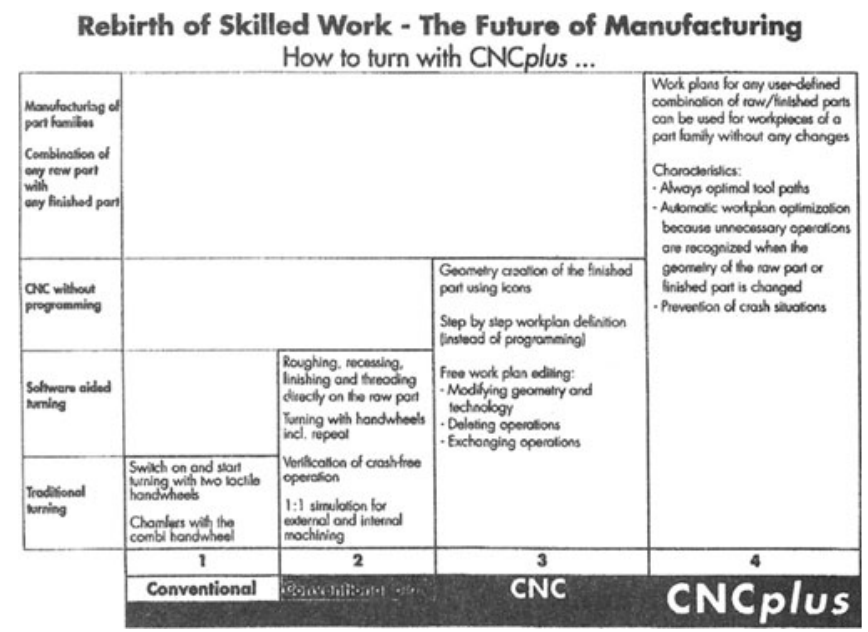

Fig. 4. The Modes of the Keller/NUM-Control

Compared to Traub this concept uses the skill of the workers in combination with intelligent software support. All kind of parts can be produced economically. It is not always necessary to use software to manufacture parts, for example in rough cutting processes or parts of a simple geometry. But if this is of advantage in respect to rationalize the process one has all the support in one and the same machine tool.

The concept MOSAIC easy of AUTOCON open systems [3] supports the skilled workers in milling and drilling operations. With the MOSAIC easy control the operators may use two operation modes: manual or pathfree. The operation mode ,manual“" provides the operation via handwheels or a joystick. The mode ,pathfree“ provides software support to define the geometry of a workpiece. The operator may switch between the modes at every time. The pathfree-software prevents the violation of the previously defined geometry when using the mode ,manual“".

This concept supports the skilled workers which mostly have a lot of experiences with conventional machine tools. In the manual mode the control works as a position indicator via the linear rules for each axis. This facilitates the manual manufacturing of parts. But the software support enables manufacturingprocesses one cannot execute with conventional machine tools. This provides maximal productivity for small batches. In addition the MOSAIC easy concept allows a more simple handling than a classic CNC-milling machine tool.

The operator may use the machine tool first in a conventional way. Then he can get software support to define a given geometry of a part from a drawing. Now he can use the handwheels or the joystick to 
start the processing. The defined geometry cannot be violated (Fig. 5). „Pathfree“ prevents it. The operator may switch to automatic processing whereby the software provides feedrates, cutting-velocity, tools and

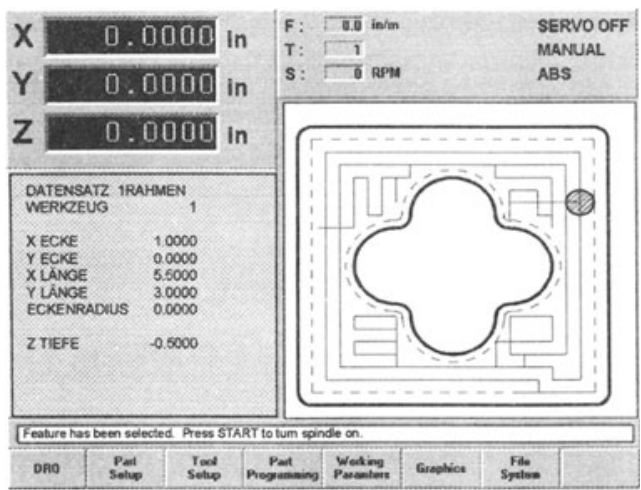

Fig. 5. The Screen of MOSAIC easy

other technological datas in respect to a certain material. The operator may accept it or overwrite it using his experiences. The use of $\mathrm{CAD}$-geometric datas from outside is possible via a network or a floppy disk. MOSAIC easy may be used in a shopfloor network as explained in Fig. 6. This network realizes some of the requirements to shopfloor oriented technologies as mentioned in the introduction of this article. The man-machine interface of the programming-software Autowop $M$ is identical with that of Autocon Mosaic at the machine control. A machine-machine interface is realized via postprocessors. CNC-programmes written at Autowop M can be used at different controls.

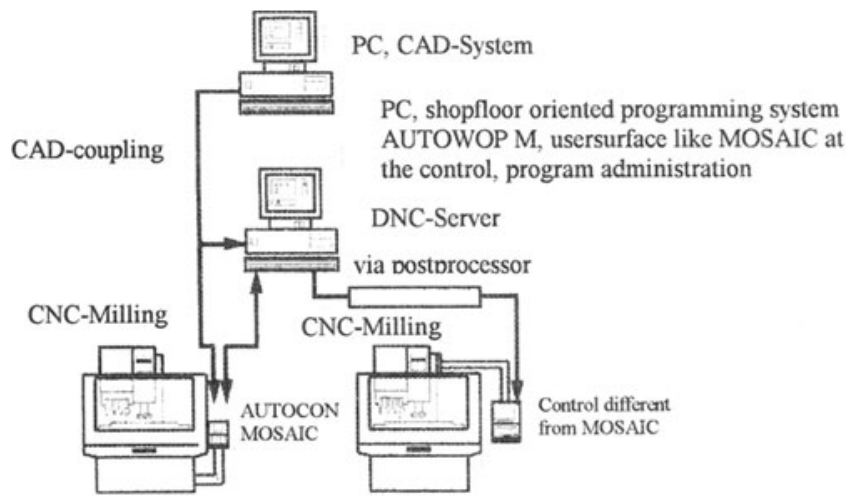

Fig. 6. Shopfloor -Network

\section{FINE PLANNING OF TASKS}

Shopfloor control is the link between the planning and administrative section of a company and the actual manufacturing process at the shopfloor. In SME's in which there are few staff to perform 
planning and setting up of tasks, shopfloor control becomes the information backbone to the entire production process.

Computerbased planning and control instruments, which are capable of working up frame datas from PPS at the shopfloor, got developed the last time(,Leitstand“). The Leitstand runs on workstations or PC's. It can be used at the shopfloor but affords specialists.

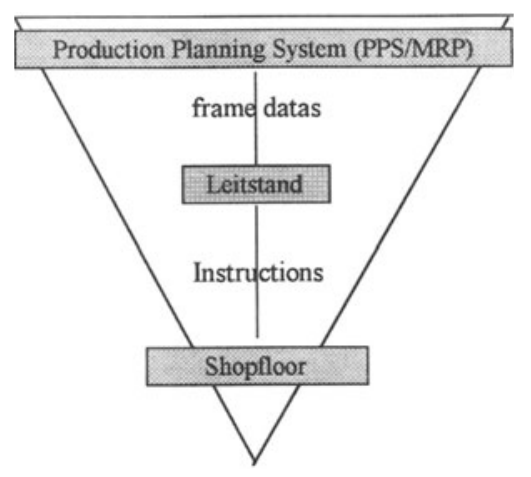

Fig. 7. Refined Top to Bottom Organization

Experiences gained when using the Leitstand in SME's have revealed last time that this instrument is not very effective. It represents a top to bottom philosophy, although refined (Fig. 7). What SME's need are low cost shopfloor control means not only to avoid more or less complicated and expensive technology in respect to investment and maintenance but it is the workorganization which should be effective.

Of course the shopfloor needs software support to enhance the flexibility and productivity. But support is to be stressed, not determination what to do by automatic decision of certain algorithm of the Leitstand.

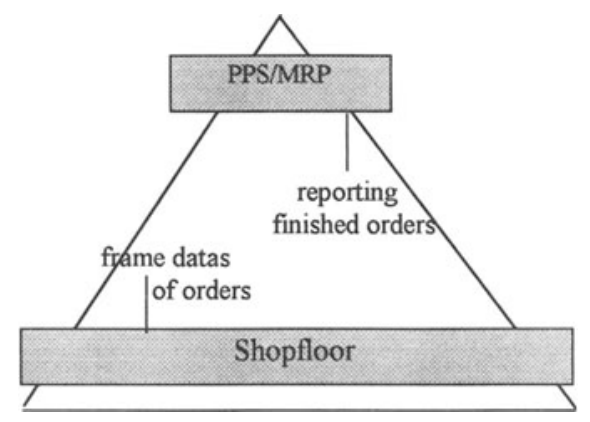

Shopfloor Control: fineplanning, disposing, scheduling of tasks belonging to orders regarding the current situation, using experiences of workers as well as recorded and evaluated process datas.

Fig. 8. Bottom to Top Organization

Today only few, if any, production planning software is available for an adequate information processing in SME's. In small batch or single production (moulds, tools, spare parts) one needs devices for a dynamic planning. Checking all solutions to the problems arising at the shopfloor, while taking 
into account all relevant restrictions, short term scheduling outside the shopfloor either by manual or automatic means is not possible.

It is without sense to schedule the manufacturing processes exactly for weeks ahead. One needs devices which are capable of calculating time corridors. The shopfloor can do the fineplanning with respect to actual circumstances much better then the central planning. Human experience regarding solutions, changing parameters and interdependencies is the very basis of shopfloor decisions and needs to be supported rather than replaced (Fig. 8).

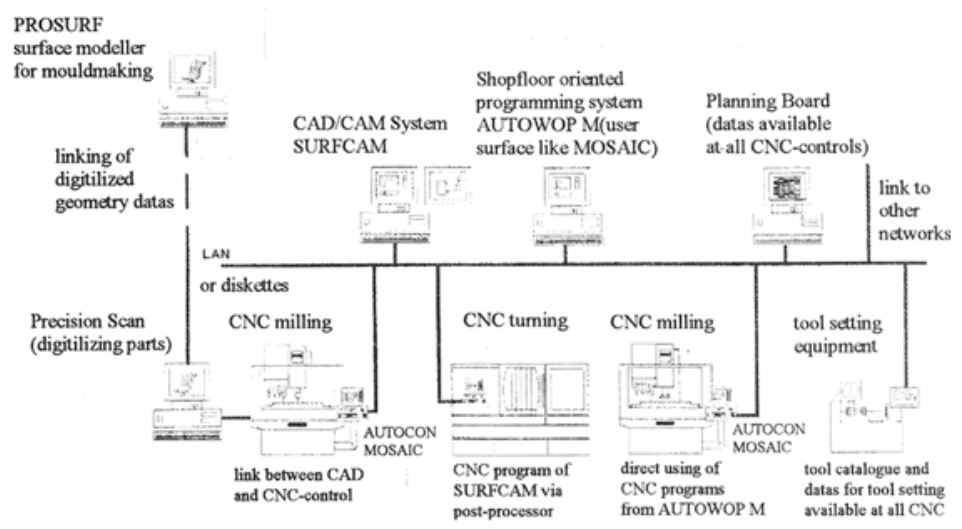

Fig. 9. Modules of a Shopfloor-Network System

Ainger [4] demonstrated that, with the help of a supporting software called ACIT (Appropriate Computer Integrated Manufacturing Technology) including a Factory Planner module and a Cell Scheduler Module running on PC's, it was possible for the workers at the shopfloor to do the order processing and scheduling of tasks. The Factory Planner module allows capacity planning for the factory as a whole without the need for detailed and time consuming scheduling of each operation and resource. This enhances visibility of the wider aspect of the production process.

The Cell Scheduler receives orders for production from the Factory Planner. Scheduling is performed by sequencing orders on each work center, either interactively or by use of one of the number algorithms provided by the system.

Fig. 9 shows a network system linking all relevant modules to be used by skilled workers at the shopfloor. It is an extension of Fig. 6. Apart from necessary devices as tool-setting and others an electronic planning board is integrated. The screen of the board is available at all CNC-controls to be used at least as an information on tasks to be done at certain workplaces to a certain schedule. As all skilled workers as a group are responsible for the manufacturing process they have beyond the access to information the task of fineplanning of orders they got with frame dates from the management. Therefore they use the electronic planning board at the CNC-controls or alternatively at the PC shown in the figure.

As was mentioned above in this paper the workers have to arrange and execute the manufacturing processes as well as the shopfloor control. Therefore the handling of the software support should be as easy as possible. Otherwise new specialists will be grown up and that is absolutely contraproductive in respect to flexibility of the SME's.

Very recently a solution was shown by Intersoft [5 ] improving decisivly ACIT by structures more suitable for the workers and supporting teamwork. Using a planning board at the PC screen based on a database the decisions of the workers in respect to shorten the through-put time or to schedule delivering can be first simulated to consider the access of tasks to available resources. So disturbances at the shopfloor (breakdown of machine tools, not just available tools, just missing personnel) can be ruled out (Fig. 10). 


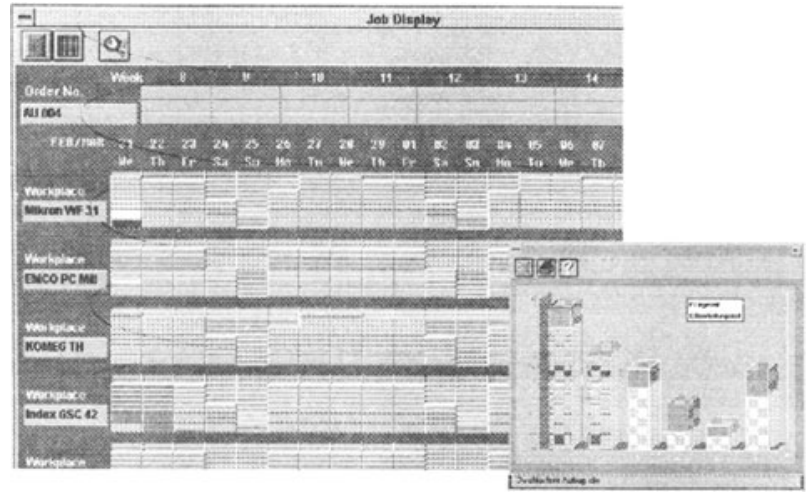

Fig. 10. Planning Board of Intersoft

\section{CONCLUSIONS: COMPUTER BASED SKILLED WORK}

Today the problem arises to foster the re-thinking of engineers and workers at the shopfloor that it is more flexible and productive to connect hands and brains more closely within the manufacturing process. This is of most relevance particularly in respect to the new paradigm of customer oriented production.

As explained in the foregoing paragraphs there are recent developments for machine tools and planning devices to support skilled workers to gain more responsibility. These developments are results of a collaboration of engineers, work-psychologists, work-pedagogics and the new busines management. The term "Computerbased skilled work" describes a new quality of work which connects informationand material-processing using these shopfloor oriented technologies.

The vocational training and education have to overcome the traditional dividing of work in planning processes as tasks of engineers or technicians outside of the shopfloor and the manufacturing process itself at machine tools, conventional workplaces or assembly-lines commanded to workers at these places.

The qualifying of workers in respect to a future development of production technologies has to foster the competence of the shopfloor as a whole.

The demands on vocational training are growing. Particularly the permanent qualifying within the work, i.e. at the workplace, needs more attention than before. The demands on training actions are of another kind than only to get a functionality of the workers. Demanded for are innovative qualifications as to organize, to supervise, to maintain the manufacturing process.

Of course this is only possible in an adequate workorganization. Groupwork or at least the co-operation of skilled workers with different basic vocational training experiences as for example turning, milling, assembling, maintaining, quality inspecting should be the backbone of the manufacturing. This supports the selfsharpening of skills and the collecting of mutual experiences.

\section{REFERENCES}

[1] M. Hekeler, Traub-IPS, Workshop oriented programming system, Preprint of Traub AG, 1989

[2] S. Keller, Von der Erfindung des Rades, Maschine und Werkzeug, Vol. 20, 1994

[3] H. Politsch, Integration der CNC-Werkzeugmaschinen in unterschiedliche betriebliche Strukturen, Preprints NC-Gesellschaft, Annual Congress, Ulm, 1995

[4] A. Ainger, Design Strategies for Human Centred Manufacturing Systems. in: P.T. Kidd, W. Karwowski (Eds.) Advances in Agile Manufacturing. Amsterdam, 1994

[5] M. Fauser, H. Seidelmeier, Fertigungsnahe Betriebsanalyse und Arbeitsorganisation, Gilching, 1995 